\title{
Second and Third Virial Coefficients for Polyisobutylene in Heptane, an Intermediate Solvent
}

\author{
Kazutomo Akasaka, Yo Nakamura, Takashi Norisuye, \\ and Akio TERAMOTo \\ Department of Macromolecular Science, Osaka University, \\ Toyonaka, Osaka 560, Japan
}

(Received July 13, 1994)

\begin{abstract}
Light scattering measurements have been made on nine polyisobutylene (PIB) fractions in heptane, an intermediate solvent, at $25^{\circ} \mathrm{C}$ to determine the second virial coefficient $A_{2}$ and the third virial coefficient $A_{3}$ as functions of weight-average molecular weight $M_{w}$ ranging from $2.7 \times 10^{4}$ to $7.8 \times 10^{6}$. For six of the fractions, $z$-average mean-square radii of gyration have also been determined. In the molecular weight range studied, $A_{2}$ and $A_{3}$ vary as $M_{w}{ }^{-0.21}$ and $M_{w}{ }^{0.58}$, respectively, and the factor $g$ defined by $A_{3} / A_{2}{ }^{2} M_{w}$ is about 0.33 almost independent of molecular weight. The data of $g$ and $\Psi$ (the interpenetration function) as functions of the cube of the radius expansion factor $\alpha_{S}$, combined with previous data in cyclohexane, a good solvent, show that the two-parameter theory breaks down unless $\alpha_{S}{ }^{3}$ is larger than about 2 for $g$ and about 5 for $\Psi$. It is concluded from the comparison of these $g$ data with the recent theory of Norisuye $e t$ al. that the failure of the two-parameter theory for $g$ at small $\alpha_{S}{ }^{3}$ is due primarily to the neglect of three-segment interactions and that the effect of chain stiffness on $g$ in heptane is of minor importance in the $M_{w}$ range studied. On the other hand, the stiffness effect on $\Psi$ for PIB is found to be remarkable even at large $\alpha_{S}{ }^{3}$. In fact, the different dependences of $\Psi$ on $\alpha_{S}{ }^{3}$ observed for heptane and cyclohexane solutions are explained semiquantitatively by the Yamakawa theory which takes account of the stiffness effect within the binary cluster approximation.

KEY WORDS Second Virial Coefficient / Third Virial Coefficient / Polyisobutylene / Excluded-Volume Effect / Expansion Factor / ThreeSegment Interaction / Light Scattering /
\end{abstract}

Recently, we investigated the third virial coefficient $A_{3}$ for polystyrene ${ }^{1,2}$ and polyisobutylene (PIB $)^{3,4}$ in both good and theta solvents by light scattering, and drew the following conclusions from experimental findings together with some theoretical calculations. $^{5,6}$ (1) The effect of three-segment interactions on $A_{3}$ is remarkable at and near the theta point $\Theta$ at which the second virial coefficient $A_{2}$ for high molecular weight $M$ vanishes, so that the binary cluster approximation to $A_{3}$ or the two-parameter theory for it at and near $\Theta$ completely breaks down. (2) In good solvents, the ternary cluster effect on the reduced third virial coefficient $g$ is appreciable only for relatively low $M$, and thus the binary cluster approximation holds for $g$ unless the radius expansion factor $\alpha_{S}\left(\equiv\left\langle S^{2}\right\rangle^{1 / 2} /\left\langle S^{2}\right\rangle_{0}^{1 / 2}\right)$ is close to unity. Here, $g$ is defined by

$$
g=A_{3} / A_{2}{ }^{2} M
$$

and $\left\langle S^{2}\right\rangle$ and $\left\langle S^{2}\right\rangle_{0}$ are the perturbed and unperturbed mean-square radii of gyration, respectively. (3) The effect of chain stiffness on $g$ in good solvents is rather small differing from the case of $A_{2},{ }^{7,8}$ though it affects the relation between $g$ and $\alpha_{S}{ }^{3}$ for small $\alpha_{S}{ }^{3}$ owing to the stiffness effect on $\alpha_{S}{ }^{9-11}$

For a deeper understanding of the ternary cluster effect on $\mathrm{A}_{3}$ we deemed it significant to explore the behavior of $g$ in a solvent intermediate between good and theta solvents, 
and undertook a light scattering study, with PIB in heptane at $25^{\circ} \mathrm{C}$ chosen as the system. According to Matsumoto et al., ${ }^{12}$ heptane is such a solvent for PIB. In the work reported below, we present experimental results for $A_{2}$, $A_{3}$, and $\left\langle S^{2}\right\rangle_{z}$ (the $z$-average mean-square radius of gyration) as functions of $M_{w}$ (the weight-average molecular weight) and compare the $g$ data with our previous data ${ }^{3}$ for the same polymer in cyclohexane, a good solvent, and also with theoretical predictions. Brief discussions on $\alpha_{S}$ and the interpenetration function appearing in $A_{2}$ are given in relation to the stiffness effect within the binary cluster approximation. We note that three-segment interactions need not be considered explicitly for these properties. ${ }^{6,13}$

\section{EXPERIMENTAL}

Nine PIB fractions ranging in $M_{w}$ from $2.7 \times 10^{4}$ to $7.8 \times 10^{6}$ were chosen from the previously investigated samples. ${ }^{3,4}$ The weightaverage to number-average molecular weight ratios of five of them were $1.08-1.10 .^{3}$ Each of the chosen fractions (originally vacuumdried or freeze-dried) was further dried in vacuo for more than one day just before use. Its mixture with heptane was stirred gently, especially for high molecular weight fractions $\left(M_{w}>3 \times 10^{6}\right)$, at room temperature for two to ten days to ensure complete dissolution. The solvent heptane was fractionally distilled over sodium after being refluxed for about $5 \mathrm{~h}$.

The polymer mass concentration $c$ of each solution was calculated from the polymer weight fraction with the solution density $\rho$. The density was measured at $25^{\circ} \mathrm{C}$ by a bicapillary pycnometer of $30 \mathrm{~cm}^{3}$ capacity. The results for $c \lesssim 4 \times 10^{-2} \mathrm{~g} \mathrm{~cm}^{-3}$ was found to be represented by

$$
\rho=0.67961+0.273 c+0.11 c^{2} \quad\left(\mathrm{~g} \mathrm{~cm}^{-3}\right)
$$

Light scattering intensities were measured at $25^{\circ} \mathrm{C}$ on a Fica-50 light-scattering photometer in an angular range from $18^{\circ}$ to $150^{\circ}$ with vertically polarized incident beam of $436 \mathrm{~nm}$ or $546 \mathrm{~nm}$ wavelength. The apparatus was calibrated with benzene at $25^{\circ} \mathrm{C}$ (see ref 14). The depolarization ratio of benzene, determined by the method of Rubingh and $\mathrm{Yu},{ }^{15}$ was 0.40 for both $436 \mathrm{~nm}$ and $546 \mathrm{~nm}$.

As in our previous work, ${ }^{1-4}$ seven different concentrations were studied for each polymer fraction. They were made optically clean by the method described elsewhere. ${ }^{14}$

The specific refractive index increment $\partial n / \partial c$ for PIB in heptane at $25^{\circ} \mathrm{C}$ was determined by using a modified Schulz-Cantow type differential refractometer. The values obtained were $0.144_{4} \mathrm{~cm}^{3} \mathrm{~g}^{-1}$ and $0.141_{4} \mathrm{~cm}^{3} \mathrm{~g}^{-1}$ for $436 \mathrm{~nm}$ and $546 \mathrm{~nm}$, respectively, independent of $c$ below $4 \times 10^{-2} \mathrm{~g} \mathrm{~cm}^{-3}$. The value for $436 \mathrm{~nm}$ agreed closely with the literature data of Matsumoto et al. ${ }^{12}\left(0.1422 \mathrm{~cm}^{3} \mathrm{~g}^{-1}\right)$ and Abe et al. ${ }^{16}\left(0.1435 \mathrm{~cm}^{3} \mathrm{~g}^{-1}\right)$.

\section{RESULTS}

\section{Data Analysis}

Figure 1 illustrates the angular dependence of $\left(K c / R_{\theta}\right)^{1 / 2}$ for a PIB fraction (S-14B) in heptane at $25^{\circ} \mathrm{C}$. Here, $K$ is the optical constant and $R_{\theta}$ is the reduced scattering intensity at a scattering angle $\theta$. The intercept of each line gives the zero-scattering angle value $\left(K c / R_{0}\right)^{1 / 2}$.

From the values for $K c / R_{0}$ obtained, a plot

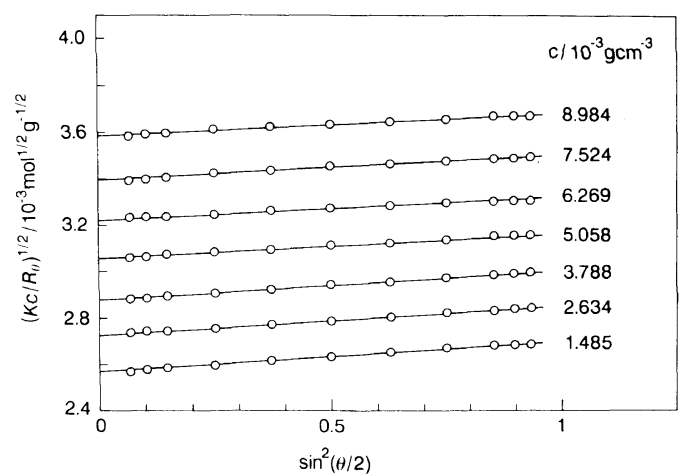

Figure 1. Angular dependence of scattering intensity at the indicated concentrations for PIB fraction S-14B in heptane at $25^{\circ} \mathrm{C}$. 

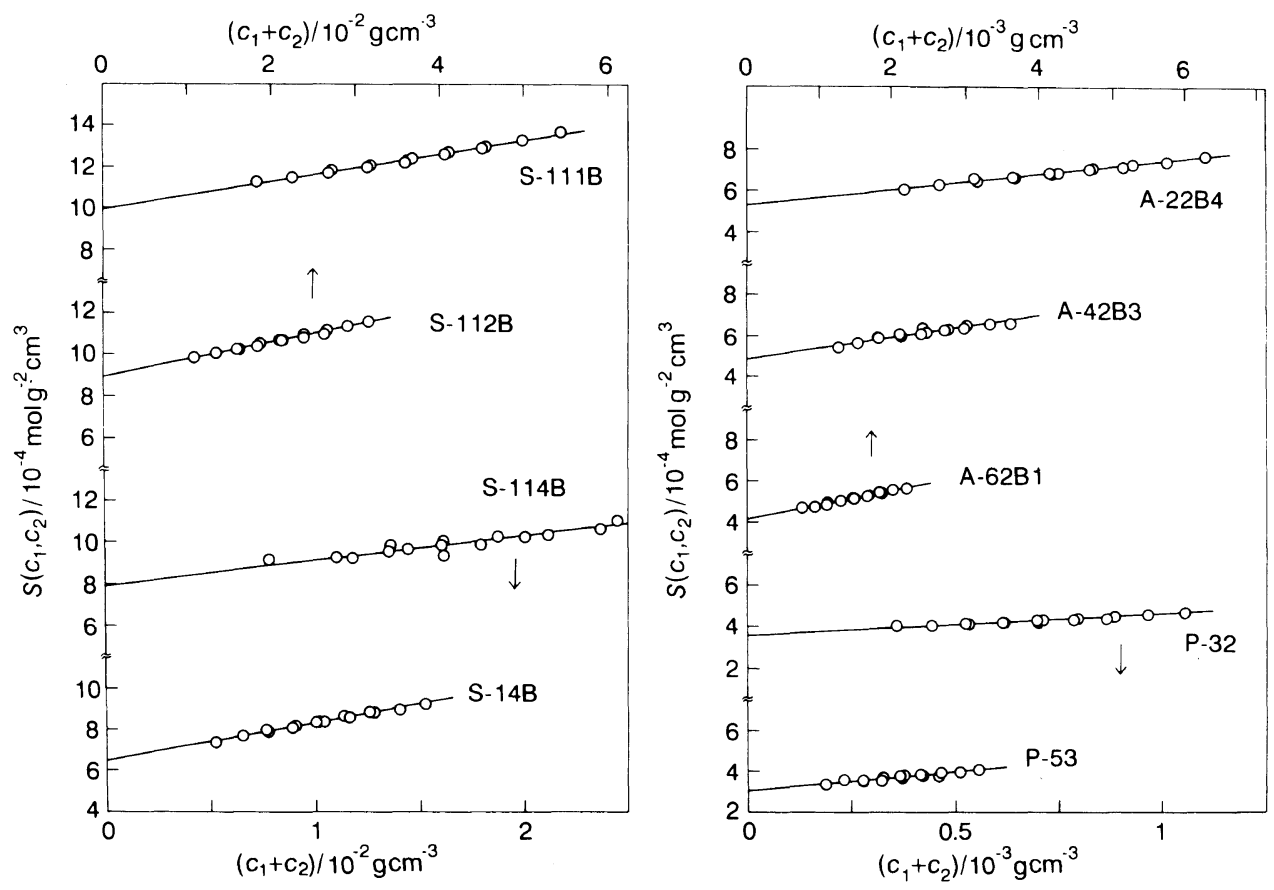

Figure 2. Plots of $S\left(c_{1}, c_{2}\right)$ vs. $c_{1}+c_{2}$ for PIB fractions in heptane at $25^{\circ} \mathrm{C} . S\left(c_{1}, c_{2}\right)$ data for pairs of neighboring $c_{1}$ and $c_{2}$ in a series of polymer concentrations are omitted, since they were not very accurate.

of $S\left(c_{1}, c_{2}\right)$ vs. $c_{1}+c_{2}$ (referred to as the Bawn plot ${ }^{17}$ ) was constructed according to the equation $^{18}$

$$
\begin{aligned}
S\left(c_{1}, c_{2}\right) & \equiv \frac{\left(K c / R_{0}\right)_{c=c_{2}}-\left(K c / R_{0}\right)_{c=c_{1}}}{c_{2}-c_{1}} \\
& =2 A_{2}+3 A_{3}\left(c_{1}+c_{2}\right)+\cdots
\end{aligned}
$$

where $c_{1}$ and $c_{2}$ denote different concentrations. The resulting Bawn plot is shown in Figure 2, along with those constructed for other fractions. The data points for each fraction are fitted by a straight line, whose intercept and slope give $2 A_{2}$ and $3 A_{3}$, respectively.

The molecular weights of all the fractions were determined as follows. ${ }^{18}$ The apparent molecular weight defined by

$$
M_{\text {app }}=\left[\left(K c / R_{0}\right)-2 A_{2} c-3 A_{3} c^{2}\right]^{-1}
$$

was calculated for each fraction with the estimated $A_{2}$ and $A_{3}$ values and plotted against $c$. As Figure 3 shows, the plotted points for any fractions fall on the respective horizontal

\begin{tabular}{|c|c|c|c|c|}
\hline \multirow{2}{*}{ Fraction } & \multirow{2}{*}{$M_{w} \times 10^{-5}$} & $A_{2} \times 10^{4}$ & $A_{3} \times 10^{2}$ & $\left\langle S^{2}\right\rangle_{z}{ }^{1 / 2}$ \\
\hline & & $\mathrm{mol} \mathrm{g}^{-2} \mathrm{~cm}^{3}$ & $\mathrm{molg}^{-3} \mathrm{~cm}^{6}$ & $\mathrm{~nm}$ \\
\hline S-111B & 0.271 & 4.96 & 0.22 & - \\
\hline S-112B & 0.425 & 4.45 & 0.28 & - \\
\hline S-114B & 0.813 & 3.94 & 0.40 & - \\
\hline S-14B & 1.79 & 3.22 & 0.62 & 15.4 \\
\hline A-22B4 & 4.87 & 2.65 & 1.21 & 27.9 \\
\hline A-42B3 & 8.90 & 2.42 & 1.73 & 39.7 \\
\hline A-62B1 & 15.8 & 2.08 & 2.3 & 55.9 \\
\hline P-32 & 33.1 & 1.78 & 3.4 & 87.1 \\
\hline P-53 & 77.5 & 1.51 & 6.1 & 145 \\
\hline
\end{tabular}

Table I. Results from light scattering measurements on PIB fractions in heptane at $25^{\circ} \mathrm{C}$

lines. The intercepts give the desired $M_{w}$.

The $z$-average mean-square radius of gyration was determined by the usual method. ${ }^{19}$ The results for $M_{w}, A_{2}, A_{3}$, and $\left\langle S^{2}\right\rangle_{z}$ are all summarized in Table I. The $M_{w}$ values for five fractions (S-114B, S-14B, A-22B4, A-42B3, A-62B1) agree with the previously determined 


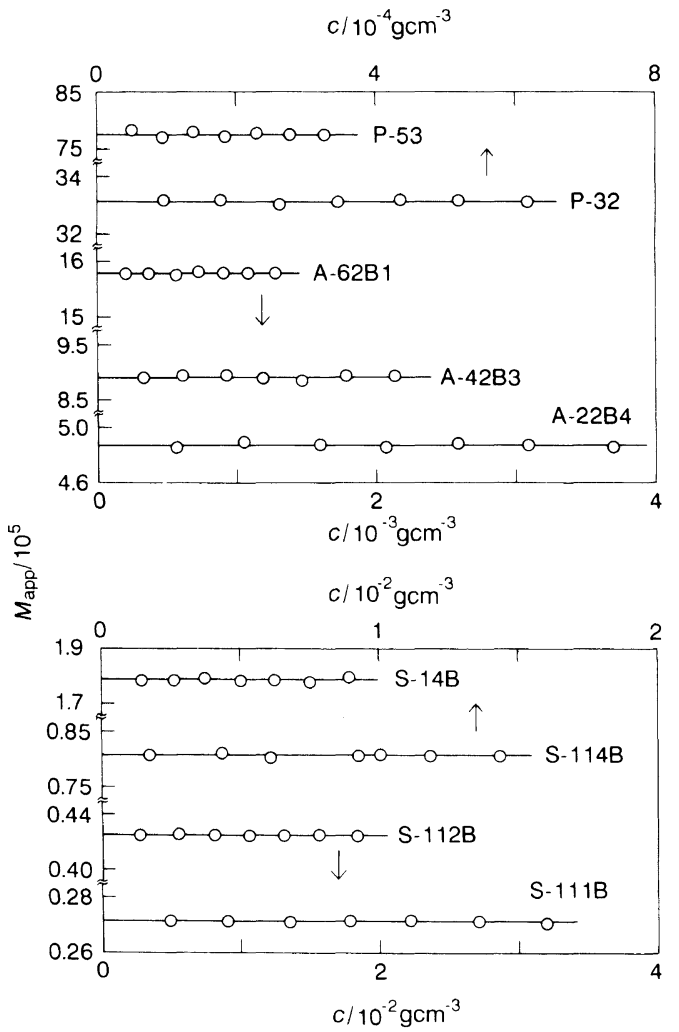

Figure 3. Plots of $M_{\text {app }} v s . c$ for PIB fractions in heptane at $25^{\circ}$ C. $M_{\text {app }}$ is defined by eq 3 .

values $^{4}$ in isoamyl isovalerate at the theta temperature $\left(27^{\circ} \mathrm{C}\right)$ within $\pm 1 \%$ and those for six fractions (S-112B, S-14B, A-22B4, A-42B3, A-62B1, P-53), with the previous values ${ }^{3}$ in cyclohexane within $\pm 1 \%$.

\section{Second and Third Virial Coefficients}

Figure 4 shows the molecular weight dependence of $A_{2}$ for PIB in heptane. It includes the data of Matsumoto et al. ${ }^{12}$ in the same solvent and those of Nakamura et ll $^{3}$ in cyclohexane $(\mathrm{CH})$, all at $25^{\circ} \mathrm{C}$. The agreement between our data and those of Matsumoto et $a l$. is very good. The straight lines fitting these two data sets has a slope of -0.21 , whose absolute value is slightly smaller than that $(-0.24)$ for $\mathrm{CH}$ solutions.

The molecular weight dependence of $A_{3}$ for heptane solutions is shown in Figure 5, along

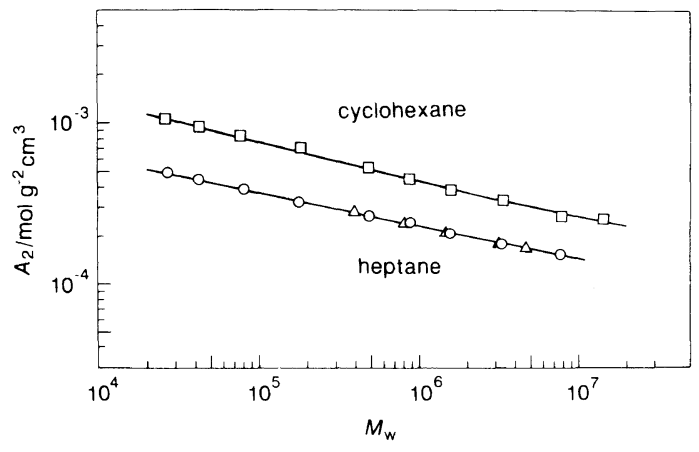

Figure 4. Molecular weight dependence of $A_{2}$ for PIB in heptane at $25^{\circ} \mathrm{C}$ (circles, this work; triangles, Matsumoto et al. ${ }^{12}$ ) and in $\mathrm{CH}$ at $25^{\circ} \mathrm{C}$ (squares, Nakamura et al. ${ }^{3}$ ).

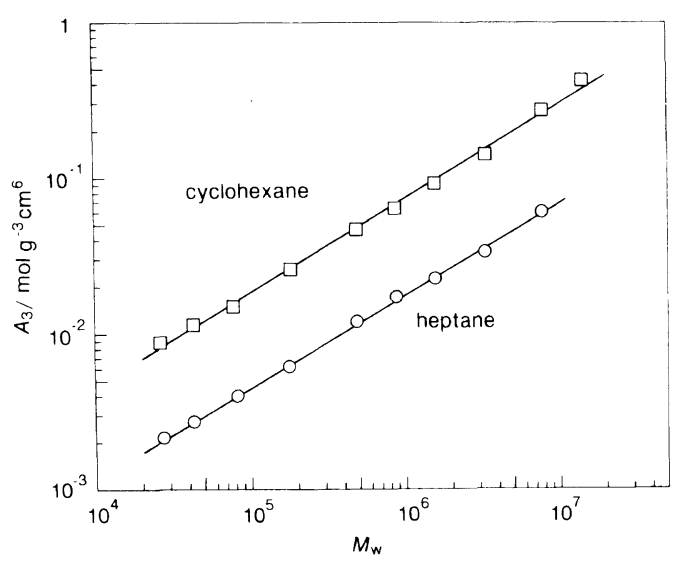

Figure 5. Molecular weight dependence of $A_{3}$ for PIB in heptane at $25^{\circ} \mathrm{C}$ (circles, this work) and in $\mathrm{CH}$ (squares, Nakamura et al. ${ }^{3}$ ), both at $25^{\circ} \mathrm{C}$.

with that determined previously ${ }^{3}$ for $\mathrm{CH}$ solutions. The $A_{3}$ values in heptane are considerably smaller than those in $\mathrm{CH}$, but the slope 0.58 of the indicated straight line for the former solvent is very close to that $(0.60)$ for the latter solvent.

In Figure 6, the values for the reduced third virial coefficient (defined by eq 1 ) in heptane are compared with those in $\mathrm{CH}$. Interestingly, the $g$ values in the former are about 0.33 , regardless of molecular weight, differing from $g$ in the latter which slightly decreases and then increases to about 0.5 with increasing $M_{w}$. The two-parameter theory ${ }^{20}$ predicts that $A_{2}$ and $A_{3}$ in the good solvent limit vary as $M_{\mathrm{w}}{ }^{-0.20}$ 


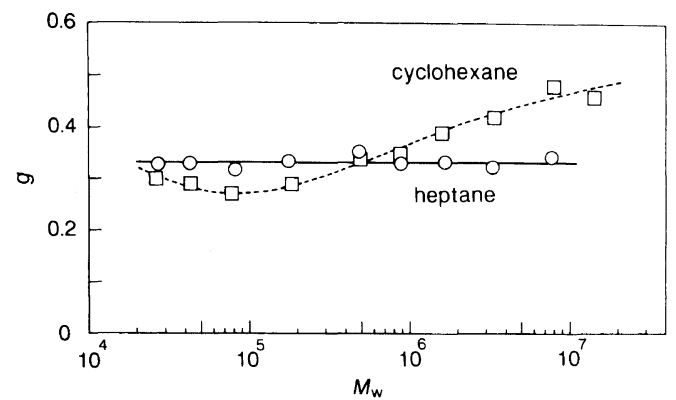

Figure 6. Molecular weight dependence of $g$ for PIB in heptane (circles, this work) and in $\mathrm{CH}$ (squares, Nakamura et al. ${ }^{3}$ ), both at $25^{\circ} \mathrm{C}$.

and $M_{w}{ }^{0.60}$, respectively. The experimental exponents of -0.21 and 0.58 for $A_{2}$ and $A_{3}$ in heptane happen to be close to these theoretical exponents and indeed lead to $M_{\mathrm{w}}$-independent $g$. On the other hand, the increase in $g$ for $\mathrm{CH}$ solutions at high $M_{w}$ is due primarily to the $M_{w}$-dependence of $A_{2}$ stronger than the two-parameter theory prediction, as discussed previously; ${ }^{3,21}$ note that the same is true for polystyrene in benzene. ${ }^{1,14}$ Since heptane is not as good a solvent for PIB as $\mathrm{CH}$, the observed $M_{w}$-independent $g$ for it should not be taken as the asymptotic behavior for flexible chains in good solvents. Another point to note in Figure 6 is that the $g$ values in heptane for $M_{w} \lesssim 5 \times 10^{5}$ are comparable to or somewhat larger than those in $\mathrm{CH}$ at the same $M_{w}$. This contradicts the two-parameter theory prediction that $g$ for a given polymer in an intermediate solvent is always smaller than that in a good solvent at the same molecular weight.

\section{Radius of Gyration}

The present $\left\langle S^{2}\right\rangle_{z}$ data in heptane (the unfilled circles) are compared with those of Matsumoto et al. ${ }^{12}$ (the triangles) and Abe et al. ${ }^{16}$ (the inverted triangles) in Figure 7 , in which our previous data in $\mathrm{CH}$ (the squares) ${ }^{3}$ and in isoamyl isovalerate (IAIV) ${ }^{4}$ at the theta temperature (the filled circles) are included. Here, one filled circle $\left(M_{w}=3.32 \times 10^{6},\left\langle S^{2}\right\rangle_{0 z}=\right.$ $\left.3.40 \times 10^{3} \mathrm{~nm}^{2}\right)$ is the data obtained for fraction $\mathrm{P}-32$ in this work. The literature data in $\mathrm{CH}$

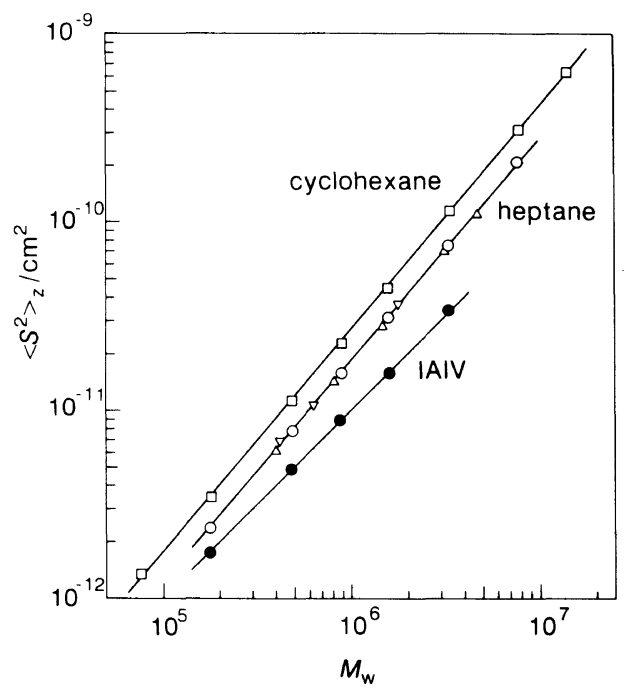

Figure 7. Molecular weight dependence of $\left\langle S^{2}\right\rangle_{z}$ for PIB in heptane at $25^{\circ} \mathrm{C}$ (unfilled circles, this work; triangles, Matsumoto et al.; ${ }^{12}$ inverted triangles, Abe et al. ${ }^{16}$ ), in $\mathrm{CH}$ at $25^{\circ} \mathrm{C}$ (squares, Nakamura et al. ${ }^{3}$ ), and in IAIV at the theta temperature $27^{\circ} \mathrm{C}$ (filled circles, Akasaka et al. ${ }^{4}$ ).

and in IAIV at the theta point by other workers $^{12,16}$ are not shown here, since they were compared with ours previously. ${ }^{3,4}$ All the data points in heptane are seen to be fitted by a single straight line. This line has a slope of 1.18 , which does not differ from the value 1.19 in $\mathrm{CH}$.

\section{DISCUSSION}

\section{Expansion Factor and Interpenetration Function}

For convenience of our discussion, we first examine the behavior of $\alpha_{S}^{2}$ in heptane and $\mathrm{CH}$, with the unperturbed PIB chain modeled by the wormlike bead chain, a special case of the helical wormlike chain. ${ }^{22}$ In the Yamakawa-Stockmayer-Shimada (YSS) theoretical scheme, ${ }^{9-11} \alpha_{S}$ is a universal function of the scaled excluded-volume parameter $\tilde{z}$ defined by

$$
\tilde{z}=\frac{3}{4} K(\lambda L) z_{2}
$$

with 


$$
z_{2}=\left(\frac{3}{2 \pi}\right)^{3 / 2} B_{2}(\lambda L)^{1 / 2}
$$

and

$$
\begin{aligned}
K(\lambda L)= & \frac{4}{3}-2.711(\lambda L)^{-1 / 2}+\frac{7}{6}(\lambda L)^{-1} \\
= & (\lambda L)^{-1 / 2} \exp \left[-6.611(\lambda L)^{-1}\right. \\
& +0.9198+0.03516(\lambda L)] \text { for } \lambda L \leq 6
\end{aligned}
$$

In these equations, $L$ is the contour length of the chain, $\lambda^{-1}$ is the stiffness parameter (equal to the Kuhn segment length in the wormlike chain limit), and $B_{2}$ is defined by $B_{2}=\beta_{2} \lambda / l^{2}$ with $\beta_{2}$ the binary cluster integral and $l$ the bead spacing; in the coil limit, $\tilde{z}$ becomes equal to the excluded-volume parameter in the two-parameter theory. ${ }^{20}$ The effect of chain stiffness on $\alpha_{S}$ for a given polymer comes from the deviation of $K(\lambda L)$ from the coil-limiting value of $4 / 3$. As mentioned in the Introduction, the ternary cluster effect, if any, is absorbed in the binary cluster term and hence does not need to be considered as far as $\alpha_{S}$ and $A_{2}$ are concerned. ${ }^{6,13}$

Assuming that the shift factor $M_{L}(=M / L)$ of the PIB chain is equal to $241 \mathrm{~nm}^{-1},{ }^{16}$ we obtain $\lambda^{-1}=1.4 \mathrm{~nm}$ from the $\left\langle S^{2}\right\rangle_{0 z}$ data in Figure 7 and the relation $\left\langle S^{2}\right\rangle_{0}=M /\left(6 \lambda M_{L}\right)$ for long wormlike chains. If these wormlike chain parameters are applied to heptane and $\mathrm{CH}$ solutions, $B_{2}$ is estimated to be 0.08 in heptane and 0.22 in $\mathrm{CH}$ from the slopes of $\left\langle S^{2}\right\rangle_{z} / M_{w}$ vs. $M_{w}{ }^{1 / 5}$ plots at high $M_{w}$ based on the asymptotic relation ${ }^{14,23} \alpha_{S}{ }^{2}=1.53 z_{2}{ }^{2 / 5}$ (see ref 24 for the procedure). With these parameter values, $\alpha_{S}{ }^{2}$ vs. $\tilde{z}$ relations were obtained from the present and literature data $^{3,4,12,16}$ for $\left\langle S^{2}\right\rangle_{z}$ and $M_{w}$.

The results are displayed in Figure 8 , in which the values of $\alpha_{S}{ }^{2}$ obtained with the aid of the experimental relation $\left\langle S^{2}\right\rangle_{0 z}=1.00 \times$ $10^{-17} M_{w} \mathrm{~cm}^{2}$ in IAIV $^{4}$ are included; they are

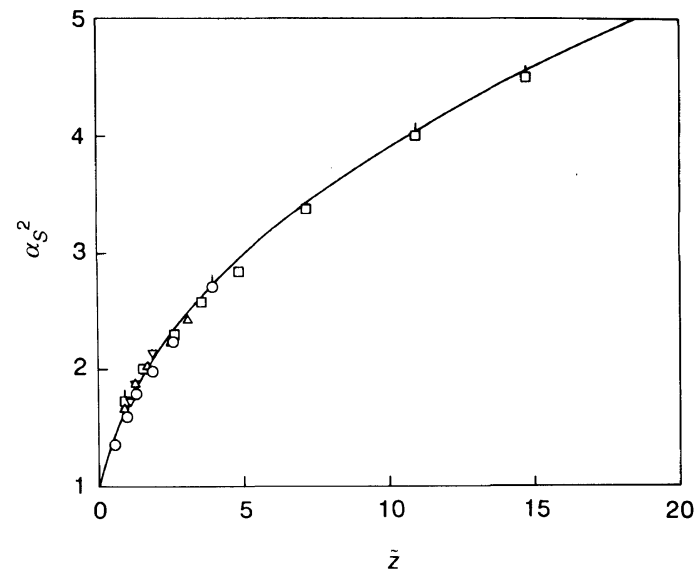

Figure 8. Plots of $\alpha_{S}{ }^{2} v s . \tilde{z}$ for PIB in heptane and in $\mathrm{CH}$ The symbols are the same as those in Figure 7 (see the text for the meaning of pips). The solid curve represents eq 7 .

one circle with pip (in heptane) and three squares with pip (in $\mathrm{CH}$ ). All the plotted points are seen to fall essentially on a single curve, being consistent with the YSS scheme or the quasi two-parameter theory. Actually, this curve represents the values calculated from the Domb-Barrett function ${ }^{25}$

$$
\begin{aligned}
a_{\mathrm{S}}{ }^{2}= & {\left[1+10 \tilde{z}+\left(\frac{70 \pi}{9}+\frac{10}{3}\right) \tilde{z}^{2}+8 \pi^{3 / 2} \tilde{z}^{3}\right]^{2 / 15} } \\
& \times\left[0.933+0.067 \exp \left(-0.85 \tilde{z}-1.39 \tilde{z}^{2}\right)\right]
\end{aligned}
$$

which reduces to the original Domb-Barrett equation in the coil limit. We note that the stiffness effect on the excluded-volume parameter amounts to $13 \%$ for the lowest-molecular weight fraction S-114B in $\mathrm{CH}$.

We next discuss the interpenetration function $\Psi$ defined by

$$
\Psi=\frac{A_{2} M^{2}}{4 \pi^{3 / 2} N_{\mathrm{A}}\left\langle S^{2}\right\rangle^{3 / 2}}
$$

where $N_{\mathrm{A}}$ is the Avogadro constant. The values of $\Psi$ in heptane calculated from the data in Table I are shown by circles in Figure 9, along with those of Matsumoto et al. ${ }^{12}$ in the same solvent (the triangles) and Nakamura et al. in 


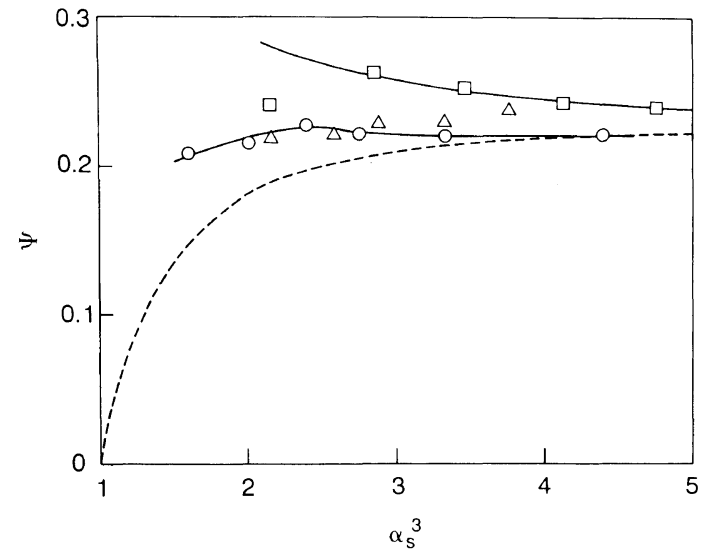

Figure 9. Plots of $\Psi$ vs. $\alpha_{S}{ }^{3}$ for PIB in heptane and in $\mathrm{CH}$. The symbols are the same as those in Figure 7. The dashed line represents the two-parameter theory values calculated from the Barrett equation. ${ }^{26}$

$\mathrm{CH}$ (the squares). Our $\Psi$ values in heptane and those of Matsumoto et al. come close to each other and are about 0.22 almost independent of $\alpha_{s}{ }^{3}$ over the molecular weight range studied. The data in $\mathrm{CH}$ appear systematically above those in heptane, revealing the breakdown of the two-parameter theory which predicts $\Psi$ to be a universal function of $\alpha_{S}{ }^{3}{ }^{20}$ As an example, the two-parameter theory values calculated from the Barrett equation ${ }^{26}$ for $\Psi\left[=\left(z_{2} / \alpha_{S}{ }^{3}\right)\left(1+14.3 z_{2}+57.3 z_{2}{ }^{2}\right)^{0.2}\right]$ and the Domb-Barrett equation for $\alpha_{S}$ (eq 7 in the coil limit) have been shown by a dashed line in the figure.

Recently, Yamakawa ${ }^{7}$ incorporated chain stiffness into $\Psi$ in the same way as that for $\alpha_{S}$ (i.e., with the helical wormlike chain), and showed that $\Psi$ is no longer a universal function of $\alpha_{S}$ but depends separately on $B_{2}$ and $M$. In particular, his theory predicts that $\Psi$ in a good solvent increases with decreasing $\alpha_{s}{ }^{3}$, while in an intermediate solvent it is systematically lower than that in the good solvent and is insensitive to $\alpha_{S}^{3}$ (see Figure 1 of ref 7). In his subsequent work, Yamakawa and collaborators ${ }^{8}$ substantiated these theoretical predictions by light scattering measurements on polystyrene in various solvents. The present data for PIB in Figure 9 also conform to the predictions, lending additional support to the validity of the Yamakawa theory. In other words, the stiffness effect on the $\Psi v s . \alpha_{S}{ }^{3}$ relation is remarkable even at $\alpha_{s}{ }^{3}$ as large as 5. It should be pointed out, however, that in order to fit the theory to the $\Psi$ data in heptane and $\mathrm{CH}$, we must choose $B_{2}$ values about three times as large as those determined above from $\alpha_{S}{ }^{2}$. As remarked by Yamakawa et al., ${ }^{8}$ the theory for $A_{2}$ is semiquantitative.

\section{Reduced Third Virial Coefficient}

In previous work, Norisuye et al. ${ }^{5}$ introduced approximately effects of chain stiffness and ternary cluster interactions into the two-parameter theory of Stockmayer and Casassa $^{27}$ for $g$. The former effect was evaluated for the wormlike bead chain in the binary cluster approximation and the latter effect, in the first-order perturbation approximation. The result is expressed by a sum of the binary cluster contribution $g_{2}$ and the ternary cluster contribution $g_{3}$, i.e.,

$$
\begin{gathered}
g=g_{2}+g_{3} \\
g_{2}=\frac{2.22 \hat{z}}{\left(1+18 \hat{z}+12.6 \hat{z}^{2}\right)^{1 / 2}} \\
g_{3}=\frac{4 B_{3}}{3 B_{2}{ }^{2} \lambda L}
\end{gathered}
$$

with

$$
\hat{z}=\left(\frac{C}{2.22}\right)\left(\frac{z_{2}}{{\alpha_{S}}^{3}}\right)
$$

Here, $B_{3}$ is the dimensionless parameter defined by $\beta_{3} \lambda^{3} / l^{3}$ with $\beta_{3}$ the ternary cluster integral for beads and $C$ is a known function of $\lambda L$ and a cutoff parameter (see Figure 1 of ref 5). In the coil limit, $C$ becomes equal to 2.22 , so that $\hat{z}$ reduces to $z_{2} / \alpha_{s}{ }^{3}$. As in our previous work, ${ }^{5}$ we take the cutoff parameter to be 0.3 .

Values of $g$ for heptane and $\mathrm{CH}$ solutions were calculated as functions of $\alpha_{S}{ }^{3}$ from the above expressions with eqs $4-7$ for the para- 


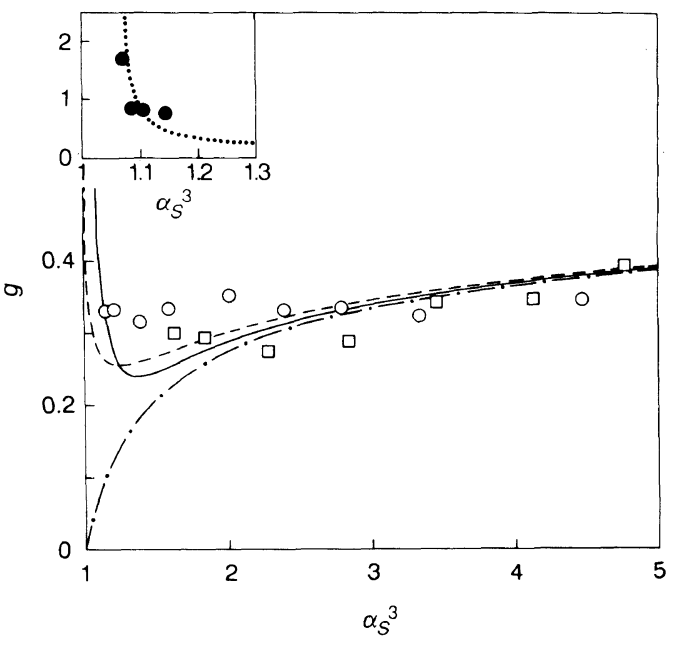

Figure 10. Comparison between experimental and theoretical values for $g$ of PIB: unfilled circles, in heptane at $25^{\circ} \mathrm{C}$; squares, in $\mathrm{CH}$ at $25^{\circ} \mathrm{C}^{3}$ filled circles, in IAIV at $37^{\circ} \mathrm{C} ;{ }^{4}$ solid line, calculated values for heptane solutions; dashed line, calculated values for $\mathrm{CH}$ solutions; dotted line, calculated values for IAIV solutions; dot-dash line, two-parameter theory values.

meter sets: $B_{2}=0.08$ for heptane, $B_{2}=0.22$ for $\mathrm{CH}$, and $M_{L}=241 \mathrm{~nm}^{-1}, \lambda^{-1}=1.4 \mathrm{~nm}$, and $B_{3}=0.021$ for both. The last parameter value was estimated from the relation $A_{3}=$ $\left(N_{\mathrm{A}}{ }^{2} / 3 M_{L}{ }^{3} \lambda^{3}\right) B_{3}$ at the theta point with the $A_{3}$ data $^{4}\left(A_{3}=5 \times 10^{-4} \mathrm{~mol} \mathrm{~g}^{-3} \mathrm{~cm}^{6}\right)$ for PIB in IAIV at $27^{\circ} \mathrm{C}$ on the assumption that $B_{3}$ is independent of solvent condition.

In Figure 10, the calculated $g$ values for heptane and $\mathrm{CH}$ solutions are shown by solid and dashed lines, respectively, in comparison with the experimental data. The dot-dash line represents the two-parameter theory values, i.e., the $g_{2}$ vs. $\alpha_{s}{ }^{3}$ relation without chain stiffness. Each of the abscissa values for a few lowest molecular weight fractions (three in heptane and two in $\mathrm{CH}$ ) is the mean of $\alpha_{S}{ }^{3}$ obtained by extrapolating the $\left\langle S^{2}\right\rangle_{z}$ data in Figure 7 and that calculated from eq 7 . Even with some ambiguity of the abscissa values for those fractions, the following points may be made from the comparison between theory and experiment.

(1) The solid or dashed line fits the data points for both heptane and $\mathrm{CH}$ solutions in the region of $\alpha_{S}{ }^{3}$ above 3 , in which the two curves almost merge with the dot-dash line. Hence the two-parameter theory holds in this $\alpha_{S}{ }^{3}$ region. (2) As $\alpha_{S}{ }^{3}$ approaches unity, the dot-dash line abruptly goes down to zero, whereas the heptane data stay almost constant. This discrepancy confirms the previous conclusion $^{5}$ (derived for good solvent systems) that the two-parameter theory breaks down for $\alpha_{S}{ }^{3}$ below 1.7. The failure of the theory in this $\alpha_{S}{ }^{3}$ region for heptane solutions is attributable primarily to the neglect of ternary cluster interactions, since the $g_{2}$ vs. $\alpha_{s}{ }^{3}$ relation with the effect of chain stiffness (not shown in the figure) comes only slightly above the dot-dash line for $\alpha_{S}{ }^{3}<2$; the stiffness effects on $g_{2}$ and $\alpha_{S}^{3}$ contribute toward lowering and raising the $g_{2}$ vs. $\alpha_{S}{ }^{3}$ curve, respectively, ${ }^{5}$ and these opposite contributions, along with the small $B_{2}$ value (see Figure 2 of ref 5), lead to the less pronounced stiffness effect on the curve for heptane. The essentially constant values of experimental $g$ in heptane (for $\alpha_{s}{ }^{3}<1.7$ ) may be explained as the result of a compensation of an increase in $g_{3}$ and a decrease in $g_{2}$ with decreasing $\alpha_{s}{ }^{3}$. (3) However, the solid curve deviates downward from the heptane data for $\alpha_{s}{ }^{3}$ between 1.2 and 2, indicating that the theory of Norisuye et al. ${ }^{5}$ with the assigned parameter values considerably underestimates $g$ for heptane solutions in the $\alpha_{S}{ }^{3}$ range. The theoretical $g$ for heptane is larger than that for $\mathrm{CH}$ below $\alpha_{\mathrm{S}}{ }^{3} \sim 1.2$, but experimentally, this occurs in a region of much larger $\alpha_{S}{ }^{3}$. Though there remains some room to adjust the $B_{3}$ values in the two solvents, the theory is not quantitative.

Because of the lack of $g$ data at low molecular weights, the present study fails to check the theoretical prediction that $g$ abruptly increases when $\alpha_{S}{ }^{3}$ approaches unity. According to eq 9 with eq 11 , however, such an abrupt increase in $g$ should be observed even in an ordinary $M_{w}$ range if the solvent conditions are made much poorer so that $B_{2}$ becomes much smaller 
(see eq 11). This is shown to be indeed the case in the insert of Figure 10, in which, as an example, the previous data $^{4}$ of $g$ for PIB fractions S-14B, A-22B4, A-42B3, and A-62B1 $\left(M_{w}=1.78 \times 10^{5}-1.60 \times 10^{6}\right)$ in IAIV at $37^{\circ} \mathrm{C}$ (the filled circles) are compared with the theoretical values (the dotted line) for $B_{2}=$ 0.004 and the same parameter values for $M_{L}$, $\lambda^{-1}$, and $B_{3}$ as those in heptane or $\mathrm{CH}$. The $B_{2}$ value has been determined so that eq 7 leads to the closest agreement with the $\alpha_{S}{ }^{2}$ data. ${ }^{4}$ As expected, the experimental $g$ values $(0.76-1.7)$ in IAIV are much larger than those in heptane. Further, their agreement with the theoretical values (the dotted line) is not bad.

\section{CONCLUSIONS}

The present data for $A_{2}$ and $A_{3}$ of PIB in heptane, when combined with the previous data in cyclohexane, give additional evidence for the breakdown of the two-parameter theory. The behavior of $\Psi$ in the two solvents can be explained semiquantitatively by the Yamakawa theory ${ }^{7}$ that takes into account the effect of chain stiffness in the binary cluster approximation. On the other hand, the binary cluster approximation with the stiffness effect does not hold for $g$ unless $\alpha_{S}{ }^{3}$ is larger than about 1.7. For $\alpha_{S}{ }^{3}<1.7$, the effect of three-segment interactions on $g$ (and hence on $A_{3}$ ) cannot be ignored, and the contribution of such interactions to $g$ increases as $\alpha_{s}{ }^{3}$ decreases or the solvent quality becomes poorer. Though qualitatively, the observed behavior of $g$ for PIB is explained by the recent theory of Norisuye et al..$^{5}$

\section{REFERENCES}

1. Y. Nakamura, T. Norisuye, and A. Teramoto, $J$.
Polym. Sci., B, Polym. Phys., 29, 153 (1991).

2. Y. Nakamura, T. Norisuye, and A. Teramoto, Macromolecules, 24, 4904 (1991).

3. Y. Nakamura, K. Akasaka, K. Katayama, T. Norisuye, and A. Teramoto, Macromolecules, 25 , 1134 (1992).

4. K. Akasaka, Y. Nakamura, T. Norisuye, and A. Teramoto, Polym. J., 26, 363 (1994).

5. T. Norisuye, Y. Nakamura, and K. Akasaka, Macromolecules, 26, 3791 (1993).

6. T. Norisuye and Y. Nakamura, Macromolecules, 27, 2054 (1994)

7. H. Yamakawa, Macromolecules, 25, 1912 (1992).

8. H. Yamakawa, F. Abe, and Y. Einaga, Macromolecules, 26, 1898 (1993).

9. H. Yamakawa and W. H. Stockmayer, J. Chem. Phys., 57, 2843 (1972).

10. H. Yamakawa and J. Shimada, J. Chem. Phys., 83, 2607 (1985)

11. J. Shimada and H. Yamakawa, J. Chem. Phys., 85, 591 (1986).

12. T. Matsumoto, N. Nishioka, and H. Fujita, J. Polym. Sci., A-2, 10, 23 (1972)

13. T. Norisuye and Y. Nakamura, Polymer, 34, 1440 (1993).

14. Y. Miyaki, Y. Einaga, and H. Fujita, Macromolecules, 11, 1180 (1978).

15. D. N. Rubingh and $\mathrm{H}$. Yu, Macromolecules, 9, 681 (1976).

16. F. Abe, Y. Einaga, and H. Yamakawa, Macromolecules, 24, 4423 (1991).

17. C. E. H. Bawn, R. F. J. Freeman, and A. R. Kamaliddin, Trans. Faraday Soc., 46, 862 (1950).

18. T. Sato, T. Norisuye, and H. Fujita, J. Polym. Sci., B, Polym. Phys., 25, 1 (1987).

19. G. C. Berry, J. Chem. Phys., 44, 4550 (1966).

20. H. Yamakawa, "Modern Theory of Polymer Solutions," Harper \& Row, New York, 1971.

21. H. Fujita and T. Norisuye, Macromolecules, 18, 1637 (1985).

22. H. Yamakawa, Ann. Rev. Phys. Chem., 35, 23 (1984).

23. M. Lax, A. J. Barrett, and C. Domb, J. Phys. A: Math. Gen., 11, 361 (1978).

24. T. Kitagawa, J. Sadanobu, and T. Norisuye, Macromolecules, 23, 602 (1990).

25. C. Domb and A. J. Barrett, Polymer, 17, 361 (1976).

26. A. J. Barrett, Macromolecules, 18, 234 (1985).

27. W. H. Stockmayer and E. F. Casassa, J. Chem. Phys., 20, 1560 (1952). 